\title{
Intermédialités
}

Histoire et théorie des arts, des lettres et des techniques

Intermediality

History and Theory of the Arts, Literature and Technologies

\section{Google : une mémoire en trompe-l'oeil}

\section{Jean-Claude Guédon}

Numéro 18, automne 2011

archiver

archiving

URI : https://id.erudit.org/iderudit/1009077ar

DOI : https://doi.org/10.7202/1009077ar

Aller au sommaire du numéro

\section{Éditeur(s)}

Revue intermédialités (Presses de l’Université de Montréal)

ISSN

1705-8546 (imprimé)

1920-3136 (numérique)

Découvrir la revue

Citer cet article

Guédon, J.-C. (2011). Google : une mémoire en trompe-l’oeil. Intermédialités /

Intermediality, (18), 121-136. https://doi.org/10.7202/1009077ar

\section{Résumé de l'article}

Cet article examine comment Google est susceptible d'affecter la mémoire. Pour ce faire, plusieurs détours sont nécessaires : après un rappel de quelques notions élémentaires sur la mémoire, nous examinerons rapidement certaines techniques de mémoire pour mieux caractériser l'écriture comme instrument de mémoire. Les supports de l'écriture retiendront ensuite notre intérêt, avec une attention particulière pour le codex qui, avec sa géométrie particulière, a dominé l'essentiel des deux derniers millénaires. Enfin, nous montrerons comment le codex donne lieu à deux formes d'analyse : la société des textes et la sociologie des textes. Tandis que la numérisation touche aux deux, Google retravaille en profondeur la société des textes, et c'est sur cette base que nous montrerons comment ce dispositif restructure profondément notre mémoire, comment il crée un trompe-l'oeil mémoriel. 


\title{
Google: une mémoire en trompe-l'œil
}

\author{
Jean-Claude Guédon \\ On est assez étonné lorsqu'on lit les traités de psychologie où il est traité \\ de la mémoire, que l'homme y soit considéré comme un être isolé.
}

Maurice Halbwachs ${ }^{1}$

\begin{abstract}
a mémoire, souvent liée exclusivement à l'individu, est pourtant et fondamentalement tributaire du collectif. Au-delà des expériences immédiates, comme la douleur par exemple, la mémoire individuelle repose sur le langage dès qu'elle doit être partagée. Or, qui dit langage, dit lente sédimentation (et érosion) d'un ensemble collectif où se love la mémoire aux côtés de toutes les formes de commerce qu'entretiennent les individus entre eux. Par ailleurs, et l'enquête policière ou le travail de l'historien le montrent bien, une forme de mémoire s'érige également au détour de procédures diverses, toutes collectives: témoignages, interrogatoires (ou interrogations) qui ont lieu sur la base de recoupements et de vérifications. De ces procédures minutieuses émerge au bout du compte un récit désormais stabilisé par l'écriture et préservé par des institutions du type archives, bibliothèques, bureaucraties. La mémoire renvoie donc bien à une réalité collective où l'on peut voir s'agréger des souvenirs individuels classés, organisés et structurés par des procédures mnémo-techniques. Ces procédures participent à la préservation des mémoires constituées et jouent donc ainsi le rôle d’une méta-mémoire: la mémoire des éléments mémoriels dignes (au nom de principes divers) d'être traités comme telle.
\end{abstract}

1. Maurice Halbwachs, Les cadres sociaux de la mémoire [1925], Paris, Albin Michel, 2001, p. VI. 


\section{DES MNÉMO-TECHNIQUES AU CODEX}

La poésie constitue un des exemples les plus anciens de procédure technique d'une mémoire collective: rythmes et rimes soutiennent en effet la mémoire individuelle du barde ou poète, et celui-ci peut ainsi réactiver régulièrement la mémoire collective en redonnant vie aux récits anciens. Ce rôle, on le sait, fut mis en question par Platon dans le dixième livre de la République, dans un passage célèbre où il fustige le poète, conteste sa place dans la Cité et donne sa forte préférence au philosophe. Eric Havelock interprète ce passage, troublant pour de nombreux lecteurs, comme le remplacement du professionnel de la mémoire orale (le barde) par le technicien de la mémoire écrite (le philosophe) ${ }^{2}$.

L'écriture permet effectivement d'extérioriser et de matérialiser la mémoire;

$\mathbf{2 2}$ il est même probable que cette fonction ait fortement motivé son invention: les tablettes d'argile et leurs inscriptions en cunéiformes recèlent des proportions importantes de listes qui soulignent clairement leur rôle d'aide-mémoire 3 . L'écriture, pourrait-on avancer, commence par un «désir de mémoire», mais ce type de motivation se retrouve dans toute technologie: par les imperfections qu'elle incorpore nécessairement - aucune technologie n'est parfaite -, elle révèle les insatisfactions rémanentes, les frustrations inévitables. La fonctionnalité d'une technologie se lit donc en creux, comme un désir imparfaitement assouvi.

Dans un passage célèbre du Phèdre de Platon, Socrate rappelle les limites de l'écriture, ses désirs latents ${ }^{4}$. Bachelard nous donne à cet égard un aphorisme utile: "La connaissance du réel, écrit-il, est une lumière qui projette toujours quelque part des ombres 5 .» L'écriture, technique de mémoire, projette aussi des ombres. L'écriture, à elle seule, ne peut donc régler la question de la mémoire; elle appelle au contraire des compléments techniques qui vont se distribuer entre sa morphologie même (syllabaires, alphabets, etc.) et ses vecteurs (tablettes de pierre, de bois, de cire, rouleau, puis codex) et qui contribuent graduellement à éclaircir la nature profonde et complexe de cette mémoire.

Les supports de l'écriture participent au travail de la mémoire. Lorsque ce support est facile à produire et peu coûteux, la mémoire collective peut se dis-

2. Eric Havelock, Preface to Plato, Cambridge (Mass.), Belknap Press of Harvard University Press, 1982.

3. Jack Goody, La raison graphique. La domestication de la pensée sauvage, Paris, Éditions de Minuit, coll. «Sens commun», 1979, chapitres 4 et 5.

4. Platon, Phèdre, trad. Mario Meunier, Paris, Payot, 1922, p. 274-275.

5. Gaston Bachelard, La formation de l'esprit scientifique: contribution à une psychanalyse de la connaissance, Paris, Vrin, 1993, p. 15. 
tribuer plus largement. Le codex offre un cas d'espèce intéressant à cet égard: il apparaît au début de notre ère et rapidement devient le véhicule privilégié des marginaux, des chrétiens en particulier. Ses origines sont obscures, parce que, produit probable d'un bricolage, d'une improvisation, il ne convenait pas aux formes nobles de l'écriture6.

L'origine humble du codex ne l'a pas empêché de modifier profondément le jeu de la mémoire en disposant l'écriture dans un dispositif d'une flexibilité de maniement inédite: alors que le rouleau est conçu pour soutenir la récitation à voix haute d'un texte déjà largement commis à la mémoire de l'orateur - on peut penser à la lecture à voix haute du rouleau antique comme l'équivalent du karaoké moderne -, le codex, lui, aide à se retrouver dans un texte, et donc à citer divers passages particuliers dans le but de les assembler autrement, selon des critères qui ont plus à voir avec les besoins du lecteur qu'avec les intentions du scribe. Ce travail du codex sur l'exercice de la mémoire s'effectue, bien évidemment, par inadvertance, par conséquence inattendue d'une topologie matérielle nouvelle; elle n'en ouvre pas moins la possibilité de la «mort du scribe » bien avant la possibilité de penser à celle de l'auteur. S'annonce ainsi le contour d'une mémoire «remix » qui va constituer le cœur d'une civilisation, la nôtre, vouée au changement permanent.

Plus fort encore, le codex invite à la consultation simultanée de plusieurs codex: le lecteur cherche à rapprocher des passages dispersés à travers divers ensembles de livres. La célèbre roue de Ramelli (Fig. 1) illustre clairement cet usage trans-textuel du codex: elle est conçue pour rompre l'isolement des textes et tisser au contraire des liens entre eux, renouant ainsi avec l'étymologie du mot «texte». En d'autres mots, la fameuse roue pointe vers un au-delà du texte, un hypertexte en somme, qui ne cessera de se déployer jusqu'au présent. En amont, la roue de Ramelli est aussi inscrite en filigrane dans l'Hexapla qu'Origène mobilisa pour établir le canon chrétien dans la première moitié du $3^{\mathrm{e}}$ siècle de notre ère ${ }^{7}$ : si l'écriture est le soutien, parfois problématique, de la mémoire, le codex est l'adjuvant de toute lecture critique; on le mobilise pour la recherche de fondations sûres, mais, du même mouvement, on ouvre la possibilité d'une

6. Colin Henderson Roberts et T. C. Skeat, The Birth of the Codex, Londres, Oxford University Press, 1987. À titre anecdotique, on peut signaler que saint Augustin, alors que le codex était largement utilisé à son époque, offre ses excuses pour une lettre rédigée en forme de codex et non de rouleau, ibid., p. 24.

7. Anthony Grafton et Megan Williams, Christianity and the Transformation of the Book: Origen, Eusebius, and the Library of Caesarea, Cambridge (Mass.), Belknap Press of Harvard University Press, 2008. 
critique interminable qui, depuis l'établissement du canon chrétien, hante et propulse la civilisation occidentale (au moins).

Le codex permet donc de penser à des familles de textes liés par des accointances de thèmes, de thèses, de sens, etc. : nommons ces phénomènes «société de textes ». Il souligne tout simplement le fait qu'un texte prend sens à partir d'une «société de textes », elle-même legs d'efforts antérieurs et collectifs de lecture; ce sens repose sur une dynamique intertextuelle que l'on pourrait aisément qualifier d'écologie où le texte citant (prédateur) croise le texte cité (proie) selon des règles dictées par des traditions (mémoire collective), par les enjeux d'une interprétation et par les logiques d'une stratégie. Se constitue ainsi un «territoire» textuel. À noter au passage que se noue alors une connivence secrète entre l'herméneute

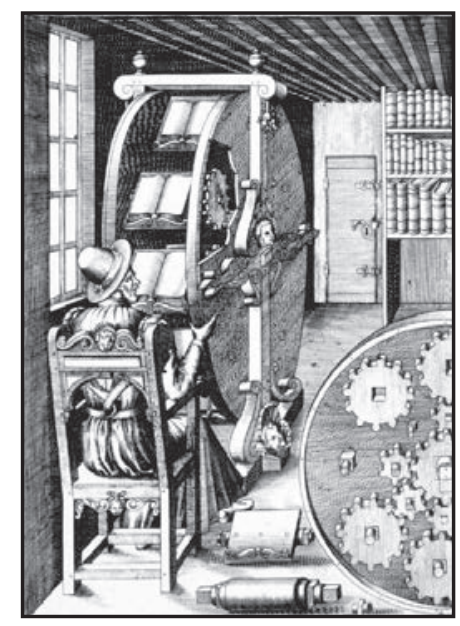

Fig. 1: Agostino Ramelli, "Roue à livres ", Le diverse et artificiose Macchine del Capitano Agostino Ramelli..., Paris, 1588, fig. 188.

et l'utopiste : chacun est marqué au coin d'une ambition commune, celle d'imposer par la raison un ordre unique, parfait, situé au-delà de toute critique possible ${ }^{8}$.

Importante certes, la dimension sociétale des textes ne suffit pourtant pas à en couvrir toute la complexité; importe aussi la trajectoire menant de la produc-

8. Rappelons que pour les géographes, ces spécialistes de l'espace de notre planète, un territoire est un espace structuré par des moyens de communication. Rappelons aussi que la Nouvelle Atlantide (New Atlantis, 1628) de Francis Bacon conçoit la cité idéale de Bensalem de façon à instituer et institutionnaliser le nouvel Organon. 
tion d'un texte à sa transmission, et finalement à sa réception et sa conservation (autre forme de mémoire collective). Elle constitue dès lors une partie du champ d'étude de la bibliographie nommée «sociologie des textes » par Donald Francis McKenzie?.

Roger Chartier, dans la préface qu'il a rédigée pour la traduction française de l'ouvrage de McKenzie, note que:

[La] bibliographie ainsi redéfinie devient une discipline centrale, essentielle pour reconstituer comment une communauté donne forme et sens à ses expériences les plus fondamentales à partir du déchiffrement des textes multiples qu'elle reçoit, produit et s'approprie ${ }^{10}$.

La description que donne Roger Chartier de la «sociologie des textes» fait écho à la description de la mémoire collective par Maurice Halbwachs. Comme lui, Chartier retrouve l'importance d'une communauté pour construire le sens. Le bibliographe ne fait que sculpter une mémoire collective appuyée sur l'écrit, et plus particulièrement sur l'imprimé; mais il le fait en parcourant la trajectoire de production et de circulation des textes et documents plutôt qu'en remplissant la carte d'un territoire textuel. Qu'est-ce qui constitue une édition? Pourquoi une deuxième édition? Questions où se croisent au moins l'économie, la culture, la connaissance et la politique. Au moins...

La roue de Ramelli pointe clairement vers ce rêve (ou désir) transtextuel que le codex porte en germe, et qui conduit également, via le «Memex » de Vannevar Bush $^{11}$, véritable miniaturisation du dispositif de Ramelli, jusqu’à la Toile ou Web. En effet, c'est le numérique qui allait véritablement propulser la société des textes au-delà de ce que le codex pouvait offrir en la matière, retrouvant une nouvelle fois l'étymologie du mot «texte».

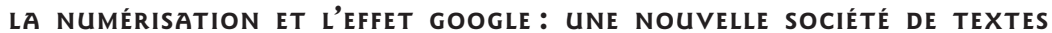

La numérisation a profondément bouleversé la sociologie des textes. Dès les années $1980^{12}$, le traitement de texte fut perçu comme radicalement neuf. On se

9. Donald Francis McKenzie, Bibliography and the Sociology of Texts [1986], Cambridge, Cambridge University Press, 1999.

10. Donald Francis McKenzie, La Bibliographie et la sociologie des textes, trad. Marc Amfreville, Paris, Cercle de la Librairie, 1991, préface de Roger Chartier, p. 9.

11. Vannevar Bush, «As We May Think», The Atlantic, juillet 1945, www.theatlantic. com/magazine/archive/1945/o7/as-we-may-think/3881/ (dernière consultation le 10 février 2012).

12. Voir Michael Heim, Electric Language: A Philosophical Study of Word Processing, New Haven, Yale University Press, 1987. 
souvient encore du sentiment de libération ressenti quand la machine se chargea enfin de gérer les notes infrapaginales automatiquement... On se souvient aussi de l'espèce d'enthousiasme qui saisissait tout un chacun à la découverte du copiercoller. Mais immédiatement de nouveaux dangers surgirent: la rédaction d'un texte, certes devenu malléable, souple, indéfiniment corrigible, menaçait aussi de devenir interminable, semblable en cela à une analyse psychanalytique... Avec le Macintosh et sa philosophie du «What you see is what you get» (Wysiwyg), la sociologie des textes a franchi une autre étape : l'ordinateur conduit à penser aussi à la présentation visuelle d'un texte, en particulier à sa typographie et à la mise en page. L'écriture d'un texte se pense désormais en relation avec son aspect visuel. Entre l'écrivain, l'éditeur et le maquettiste, les frontières s'estompent.

La sociologie des textes a donc été bouleversée par le numérique, en particulier en ce qui concerne l'aspect distribution. Au total, l'irruption de l'Internet dans le grand public au cours des années 1990 contribua à transformer la distribution en une question d'accès, mais avant même Internet, divers bricolages télématiques, y compris le fax, permettaient l'envoi de documents entre individus. La numérisation mettait ainsi à jour un désir profond et puissant de libérer les documents de toute contrainte physique ${ }^{13}$.

L'imprimeur et le typographe virent leurs métiers pris en écharpe par le numérique : avec l'avènement de l'imprimante laser, le monde des petites imprimeries se vit bousculer par une concurrence inattendue. D’autres maillons de la chaîne de production allaient aussi devoir s'adapter à ce cadre technologique nouveau. Typographie et mise en page s'effectuent maintenant grâce à des logiciels spécialisés qu'un amateur peut maîtriser en quelques heures. Photographie, son et vidéo s'ajoutent rapidement à une gamme de possibilités qui forcent à repenser le texte sous la forme plus vaste de «document».

La sociologie des textes, de la production à la réception, en passant par la transmission, a donc été totalement transformée par l'irruption du numérique: en témoignent l'ensemble des guerres - le mot n'est pas trop fort - accompagnant actuellement le droit d'auteur, le droit des marques et les brevets: elles marquent la tentative, probablement vaine, de retrouver par le biais de la loi ce qui a été perdu à cause de la technologie. Au $18^{\mathrm{e}}$ siècle, les nobles du royaume de France ont vécu une situation analogue. On nomme cette phase de la fin de

13. John Quarterman, The Matrix: Computer Networks and Conferencing Systems Worldwide, Bedford, Digital Press, 1990. Cet ouvrage dresse un portrait fascinant des réseaux télématiques à un moment où Internet ne constituait encore qu'une option parmi bien d'autres dans la gamme des technologies disponibles. 
l'Ancien Régime la «réaction nobiliaire » et on la considère souvent comme une des causes de la Révolution française...

L'écriture, ce «désir de mémoire », est elle aussi bousculée par le numérique; la mémoire collective, en particulier, se voit hantée par une nouvelle anxiété, celle de la préservation. En parallèle, la numérisation du codex engendre ce que l'on pourrait appeler une hyper-hypertextualisation du texte: le Web révèle clairement que les liens entre documents peuvent désormais se tisser à l'infini. En fait, le lien prend le dessus sur le texte: on saute d'un texte à un autre en prenant à peine le temps de lire posément la cible atteinte. On «surfe » sur des vagues de connaissances qui déferlent sur des plages d’ignorance. Mais si l'émergence d'une hyper-société des textes conduit bien évidemment à une refonte de la mémoire collective, les nouveaux contours de celle-ci sont beaucoup plus difficiles à préciser. C'est ici qu'interviennent les outils de recherche, ces lointains descendants des catalogues et autres bibliographies.

Dès le début des années 1990, Internet s'est heurté à un problème de fond: les sites FTP («file transfer protocol») s'étaient fortement multipliés bien avant l'invention du World Wide Web par Tim Berners-Lee, et son expansion explosive après 1994. Dès les années 1980, Internet ressemblait déjà à une énorme pile de documents sans catalogue. Des listes incomplètes circulaient plus ou moins sur des sujets particuliers, mais, sauf à entrer dans les bons cercles d'initiés, l'utilisateur ne pouvait que rater la plupart des ressources disponibles. Curieusement, un érudit du Moyen-Âge aurait pu se sentir à l'aise dans ce chaos de ressources dispersées, car lui aussi errait de monastère en monastère, en quête du manuscrit rêvé, et guidé par des informations et rumeurs généralement invérifiables. Dans le cas d'Internet, cette frustration a conduit à la notion de «moteur de recherche» dont «Archie» constitue une des premières formes repérables: il s'agit d'un algorithme mis au point par trois étudiants de l'Université McGill à Montréal : Alan Emtage, Bill Heelan, et J. Peter Deutsch ${ }^{14}$ en 1990.

Seulement sept ans et quelques moteurs de recherche plus tard, on passait de cet outil primitif à la fondation de Google par Larry Page et Sergey Brin ${ }^{15}$. Entretemps, le World Wide Web ${ }^{16}$ était devenu la norme de publication sur Internet,

14. Voir http://en.wikipedia.org/wiki/Archie_search_engine (dernière consultation le 10 février 2012).

15. Lee Underwood, «A Brief History of Search Engines », http://www.webreference. com/authoring/search_history/ (dernière consultation le 10 février 2012).

16. Voir http://en.wikipedia.org/wiki/World_wide_web (dernière consultation le 10 février 2012). 
et la cible privilégiée des moteurs de recherche ${ }^{17}$. Grâce à cette combinaison de la Toile et des premiers moteurs de recherche, Internet prenait la forme d'une véritable bibliothèque, dotée d'un catalogue.

Google transforme la société des textes de fond en comble en reprenant à son compte une stratégie qui avait été élaborée au cours des années 1960 par Eugene Garfield, le créateur du «Science Citation Index ${ }^{18}$ dans le domaine des bibliographies scientifiques. Garfield faisait face aux défis de l'interdisciplinarité dans les sciences de la vie. Alors que biologies moléculaire et génétique occupaient de plus en plus le devant de la scène, les bibliographies classiques continuaient de tracer les mêmes vieilles frontières disciplinaires entre chimie organique et biologie; de nombreux chercheurs, tel Joshua Lederberg, se plaignaient amèrement de ne pouvoir trouver aisément les recherches sur lesquelles ils désiraient s'appuyer. C'est dans ce contexte que Garfield a commencé à imaginer un index universel de la science en pensant d'ailleurs au «World Brain» de H. G. Wells ${ }^{19}$, mais en s'inspirant aussi de l'exemple de Shepard's, la maison d'édition qui publiait des index de jurisprudence dans le domaine du droit ${ }^{20}$. En remontant la piste des citations, imagina-t-il, on retrouve les «épaules des géants » et on les retrouve d'une société de textes qui correspondrait à l'ordre d'une histoire des sciences idéales, et non dans le cadre artificiel d'un ordre disciplinaire. La science, en d'autres termes, ne prolifère pas selon des règles de classification préétablies, mais bien en tissant de plus en plus densément une toile de connaissances, un «Web of Science » pour reprendre le nom de l'entreprise qui a racheté le Science Citation Index. Le texte scientifique, lui aussi, relève d'un désir de tisser...

Brin et Page, les deux fondateurs de Google, ont cherché à identifier les liens qui unissent les documents de la Toile entre eux en les traitant à la manière de citations. À l’instar de Garfield, ils ont également cherché à donner un sens qualitatif au nombre de ces liens pour organiser les résultats des recherches sur la Toile.

17. Tim Berners-Lee et Mark Fischetti, Weaving the Web: The Original Design and Ultimate Destiny of the World Wide Web, Londres, New York, Harper Collins, 2000.

18. Paul Wouters, "The Citation Culture », thèse de doctorat, Universiteit van Amsterdam, 1999. Voir aussi Blaise Cronin et Helen Barsky Atkins (dir.), The Web of Knowledge: A Festschrift in Honour of Eugene Garfield, Medford, Information Today, 2000.

19. Voir https://sherlock.ischool.berkeley.edu/wells/world_brain.html (dernière consultation le 10 février 2012). La vision de Wells converge aisément avec l'idée du «Memex » chez Vannevar Bush, même si ce dernier ne cite pas Wells dans son célèbre article de 1945 cité plus haut. Wouters, 1999, p. 39, donne la citation où Garfield mentionne Wells.

20. Arnold Thackray et David C. Brock, «Eugene Garfield: History, Scientific Information, and Chemical Endeavor», dans Cronin et Barsky, 2000, p. 16-17. 
Telle est, en très gros traits, la stratégie qui sous-tend le «Page Rank» de Google. Le nombre de liens visant une cible donnée renvoie à une certaine notion de visibilité et/ou de popularité (et peut-être aussi de qualité), tout comme le font les citations dans les articles scientifiques. Google s'appuie sur ces liens pour proposer des cibles potentiellement utiles correspondant aux termes d'une recherche.

De la stratégie de Google, on peut souligner quelques traits saillants :

- Google suit à la trace les liens entre documents;

- Google suit aussi les questions que posent les individus partout dans le monde et les analyse selon leur fréquence, leur origine, la durée et l'intensité de l'intérêt qui les accompagne, etc.;

- Le plan d'affaires de Google est bien connu : questions et réponses choisies suggèrent aussi les services et marchandises que des entreprises peuvent offrir.

Trop bref, ce rappel des fondements conceptuels de Google permet néanmoins d'en déduire quelques conséquences. Premièrement, le nombre de liens pointant vers une cible constitue un reflet ambigu de diverses caractéristiques - qualité, popularité, visibilité d'un site -, mais il peut aussi refléter des formes de manipulation intelligente des liens pour créer artificiellement des points de visibilité dans la Toile ${ }^{21}$. Nous sommes ici au cœur d'une économie de l'attention, c'est-à-dire d'un raisonnement économique qui fait de l'attention d'un individu, et non de la quantité d'information disponible, la denrée rare ${ }^{22}$. En même temps, ce classement contribue à créer une hiérarchie de sites, et à établir les règles et conditions d'une concurrence entre eux, concurrence de facto gérée par les algorithmes de Google. Dans le firmament de Google, certains sites ciblés par un grand nombre de liens brillent plus que d'autres, ouvrant ainsi la perspective d'une nouvelle société des textes et même des documents.

Deuxièmement, l'ensemble des liens qui pointent vers un site sur la Web proviennent d'autres sites qui, pour la plupart, n’appartiennent pas aux propriétaires du site visé. Cette collection de liens renvoie donc implicitement à une com-

21. Les algorithmes de Google tentent de neutraliser les stratégies visant à avantager certains sites en manipulant le «Page Rank» qui est l'algorithme fondamental. Le «Page Rank», incidemment, est nommé d'après Larry Page, l'un des deux fondateurs de Google. Le jeu de mots est évident. On en trouve une bonne présentation dans Wikipedia, http:// en.wikipedia.org/wiki/PageRank (dernière consultation le 10 février 2012).

22. H. A. Simon, «Designing Organizations for an Information-Rich World», dans Martin Greenberger, Computers, Communication, and the Public Interest, Baltimore, Johns Hopkins University Press, 1971, p. 40-41, cité dans l'article Wikipedia «Attention economy», http://en.wikipedia.org/wiki/Attention_economy (dernière consultation le 10 février 2012). 
munauté d'individus, mais une communauté d'une qualité particulière: le fait de connaître quelqu'un dans ce type de groupe ne garantit en rien la possibilité de connaître d'autres membres de la communauté. Inversement, un ensemble de sites peuvent se regrouper pour former une sorte «d'attracteur d'attention » en renvoyant exclusivement aux autres membres de l'ensemble. Toute personne entrant dans un de ces sites aura tendance à errer indéfiniment parmi les sites de l'«attracteur» s'il ne fait que cliquer les liens disponibles. La mémoire collective qui émerge de ces cas de figure repose sur des communautés d'un nouveau genre, agrégats d'individus constitués selon des logiques qui n'entretiennent qu'un rapport lointain avec des communautés plus traditionnelles, telle celle des lecteurs d'un livre. Ces nouveaux agrégats d'individus seront beaucoup plus dispersés que les communautés liées aux médias du passé. Ils sont souvent fondés sur un simple intérêt commun pour des documents divers (textes bien sûr, mais aussi photos et musique). Aux débuts de la Toile, les sites pornographiques, par exemple, usaient $\mathrm{d}^{\prime}$ «attracteurs » pour essentiellement retenir le chaland potentiel en lui offrant les mêmes produits «empaquetés » (si l'on peut dire...) de mille façons différentes.

De plus, sauf à sélectionner le coup de chance proposé par Google, la réponse à toute requête produit une liste, parfois immense, de réponses. Le demandeur balaie rapidement les premiers éléments de cette liste pour, du bruit ambiant, tenter d'extraire une information utile. Cette liste constitue, de facto, une société de textes, mais une «société » produite par l'interaction entre un algorithme inconnu et une requête comportant souvent divers degrés d'ambiguïté. Google, pourrait-on plaisanter, offre essentiellement une réponse «oulipienne » à nos questions, mais ici, la dimension ludique et subversive de l'Oulipo a cédé sa place à un oracle généralement pris très au sérieux ${ }^{23}$, particulièrement par les spécialistes en marketing embusqués dans les marges de Google.

Enfin, au-delà de sa capacité à classer des documents et à les associer par des procédures algorithmiques, Google a également cherché à constamment raccourcir le temps de réponse. Si je tape «Obama», j’obtiens, me dit-on, environ (!) 686 millions de réponses en o,17 seconde. Ce temps de réaction est de l'ordre de grandeur des réflexes humains, donnant ainsi l'illusion d'une réponse instantanée. Or, dans la perception et le jugement humains, le temps de réponse

23. Il existe bien des manières de décrisper le sérieux de Google, évidemment, tel le jeu du «Googlewhack». Sur celui-ci, voir http://en.wikipedia.org/wiki/Googlewhack (dernière consultation le 10 février 2012) ainsi que Jean-Claude Guédon, «Les joies du Googlewhack», La recherche, Paris, $n^{\circ}$ 352, avril 2002, p. 59. Pour ce qu'est l'Oulipo, voir www.oulipo.net/oulipiens/O (dernière consultation le 10 février 2012). 
est lié de manière subtile à la crédibilité et à l'autorité de la réponse : répondre sans hésitation connote ou bien une grande maîtrise du sujet couvert, ou une grande franchise. Si l'on ajoute à cela les algorithmes utilisés par Google pour tenter d'anticiper la fin d'une question, on bascule carrément dans le domaine de la magie: l'oracle se fait divin en quelque sorte. On attend le miracle, un peu secoué néanmoins par tant de perspicacité machinique. On ne remet pas en question une réponse de Google; on l'accueille au contraire comme un cadeau, reconnaissant de tant de mansuétude et générosité. En somme, le problème que l'écriture avait cherché à résoudre en recueillant des connaissances et en les préservant sous une forme matérielle adéquate voit maintenant une solution (demeurée incomplète avec le codex et les bibliothèques) atteindre une sorte de perfection apparente: on peut retrouver tout, n’importe où, instantanément. De l'incipit à la bibliothèque s'ébauche donc une ligne ascendante où Google culmine à une altitude inespérée. Pour la recherche d'information, Google nous fait passer à la vitesse supérieure; un nouveau paradigme s'est établi, qui poursuit néanmoins ce désir de mémoire présent dès le début de l'histoire de l'écriture.

\section{UNE MÉMOIRE EN TROMPE-L'đEIL}

Revenons à Platon : la mémoire gérée par l'écriture, «cadeau » du dieu Thot, ne plaît guère au roi Thamus. En effet, celui-ci pense que:

- l'écriture instaure un régime de mémoire qui ne vient plus des êtres humains eux-mêmes, mais de l'extérieur;

- l'écriture ne conserve que des souvenirs au mieux; la mémoire, ancrée dans les êtres, se trouve en situation de concurrence avec les souvenirs de l'écriture;

- l'écriture crée une illusion de science et laisse imaginer un apprentissage sans maître qui ne peut engendrer que de faux savants ${ }^{24}$.

Google, curieusement, offre la possibilité de réitérer ces critiques en les transposant légèrement:

- Google extériorise la mémoire, et privilégie la dimension factuelle de l'information ${ }^{25}$;

24. Voir la citation du Phèdre de Platon indiquée à la note 6.

25. B. J. Sparrow et D. M. Wegner, «Google Effects on Memory: Cognitive Consequences of Having Information at Our Fingertips », Science, 5 août 2011, n 333, vol. 6043, p. 776-778, www.sciencemag.org/cgi/doi/10.1126/science.1207745, (dernière consultation le 10 février 2012). 
- Les réponses de Google, tout comme celles de l'écriture, négligent la mémoire, entendue comme narration portant un sens, au bénéfice de souvenirs fragmentaires;

- Google, à l'instar d'une béquille, donne l'illusion d'une mémoire sans défaillance, tout en créant un régime de dépendance qui se marque par le privilège accordé aux souvenirs (faits) au détriment des arguments.

En première lecture, Google réitère et prolonge les faiblesses de l'écriture telles que décrites par le Socrate de Platon. Pour autant, il faut aussi rappeler l'autre jugement, beaucoup plus positif, de Platon sur l'écriture, situé à la fin de la République, où l'organisation politique d'une communauté ne peut se satisfaire d'une mémoire collective simplement gérée par le barde-poète. Comment résoudre cette tension entre ces deux textes de Platon?

L'écriture a probablement contribué à affaiblir certaines facultés mémorielles chez beaucoup d'individus, mais il faut pousser l'analyse plus loin. Une fois disposée dans la topologie complexe du codex et ordonnée par elle, l'écriture a aussi conduit à produire ce mouvement critique qu'inaugure à son insu Origène. Elle a donc également permis l'établissement de dialogues, discussions, débats et controverses dans sa relation à une mémoire non pas individuelle, mais collective, et une mémoire qui, de surcroît, permet également de dépasser les limites de l'espace et du temps: des traditions artistiques, philosophiques, légales, scientifiques et littéraires peuvent mourir, mais si elles ont été transcrites dans un document écrit, elles demeurent prêtes à reprendre vie au moindre souffle d'un lecteur un peu attentif. Interpellées par des textes provenant du passé, de nombreuses communautés humaines, que l'on appelle souvent des religions, contribuent à les maintenir en vie. Inversement, les textes sans lecteurs sont aussi des textes orphelins, sans protecteurs, en danger de disparition. Mais le balancier peut repartir dans une autre direction : les divisions entre des églises qui pourtant partagent les mêmes textes fondateurs rappellent les faiblesses de l'écriture pointées par Socrate. L'écriture offre ainsi, d'une part, l'occasion d'une négociation complexe entre la mémoire et toutes ses dimensions narrées, argumentatives, idéelles, conceptuelles et, d'autre part, souvenirs ponctuels, factuels, proches des choses et des actes. L'écriture s'établit également en condition de possibilité pour le maintien et l'invention de diverses formes de sociétés de textes qui s'établissent plus ou moins bien en fonction des conditions, techniques, sociales, culturelles et économiques, qui informent les pratiques de la sociologie des textes.

Au total, la lecture individuelle ne peut asseoir une mémoire forte; cependant, reprise dans un dialogue entre contemporains et non-contemporains, 
réunis par une communauté, ces mêmes textes peuvent très bien relancer le travail interne, intelligent, d'une appropriation forte par une mémoire collective dont les contours commencent dès lors à fortement ressembler à ceux d'une identité collective.

Dans cette tension entre l'individuel et le collectif, que dire de Google? Le Page Rank conduit à une forme de mémoire individuelle qui aura tendance à se modeler sur les tendances dominantes du moment, tout en privilégiant probablement ce qui se conforme le mieux à la croyance de départ de l'individu. En d'autres mots, la recherche dans Google devrait régulièrement renforcer les croyances acquises, surtout quand elles peuvent s'harmoniser avec un courant de pensée bien identifié dans la société du moment. Le résultat correspond assez bien aux effets d'un moyen de communication de masse, à ce détail près que la ligne éditoriale ne vient d'aucune conscience mais provient simplement d'effets statistiques de type émergent ou autopoiétique. La société des textes qui se construit ainsi tend à privilégier le fait, la donnée plutôt qu'une ligne interprétative. Google parle en mots, et non en paragraphes. Voilà pourquoi Google se limite à des souvenirs; ceux-ci sont issus d'un collectif, reflètent un certain degré de conformisme, mais ils ne circulent effectivement qu'entre le dispositif informatique lui-même et les individus. Cette situation explique peut-être deux phénomènes qui ont suivi la montée de Google: d'une part, les individus ont vite adopté des outils dits de «réseaux sociaux» qui leur permettaient de se constituer en communautés, à partir desquelles l'interprétation des textes placés en société par Google pouvait commencer. D’autre part, ces outils de réseaux sociaux, tel Facebook, ou Twitter, ou, plus récemment, "Google + », se sont révélés être eux aussi de puissants moteurs de recherche, fondés cette fois non sur des algorithmes, mais bien sur des communautés humaines et leurs interrogations, questions, débats. Google + était, pourrait-on dire, inévitable dans la mesure où ce nouvel outil complète de manière essentielle le moteur de recherche en facilitant la possibilité pour chaque individu de renforcer sa mémoire personnelle, individuelle, alimentée par Google, par l'adjonction d'une mémoire collective fondée sur l'adhésion à telle ou telle communauté d'utilisateurs.

Un dernier point, celui de l'oubli, permet d'approcher plus près du type de mémoire que propose Google. Diverses anecdotes, plus ou moins cruelles, décrivent ainsi des individus rattrapés par leur passé de manière inattendue: une soirée un peu trop débridée, capturée trop facilement par des appareils photo trop disponibles, nous renvoie non seulement à une nouvelle sociologie du document, mais aussi à la construction d'une mémoire peut-être trop riche et 
trop détaillée pour nos besoins réels ${ }^{26}$. La richesse même de cette mémoire peut engendrer d'autres effets plus subtils. Rappelons que l'œil capture une image de haute résolution, en gros l'équivalent de 15 mégapixels, plusieurs dizaines de fois par seconde. Si nous nous souvenions de toutes ces images, le cerveau serait vite submergé, sa capacité de mémoire se révélant à l'évidence insuffisante. Le cerveau filtre et classe avant de placer quoi que ce soit dans la mémoire à long terme de l'individu. De façon inconsciente, le cerveau travaille donc à oublier une immense partie de ce qui nous touche ${ }^{27}$. Plus important encore, si nous perdions cette capacité d'oublier, nous pourrions nous retrouver dans la position du personnage Funes de Borges: une mémoire parfaite l'empêche de généraliser et d'abstraire ${ }^{28}$. Ainsi que le résume Mayer-Schönberger :

Using generalizations, relying on conjectures, emphasizing the present, and respecting subsequent experiences, helps us to reason swiftly and economically, to abstract and generalize, and to act in time, rather than remain caught up in conflicting recollections ${ }^{29}$.

Ces remarques appliquées à la mémoire nous permettent de nous demander si ce que nous propose Google ne serait pas justement une forme de mémoire apparemment parfaite dont pourrait rêver l'ingénieur cherchant à indexer l'ensemble de l'univers documentaire? Et, si oui, cela ne reviendrait-il pas à nous limiter à une mémoire factuelle, incapable de construire du sens? De plus, ces «faits » ne sont pas validés de façon évidente ou simple; données sous forme fragmentaire, les réponses de Google peuvent fort bien ne véhiculer qu'une rumeur, une légende, mais auréolées d'une certaine autorité et véracité qui peuvent aider à emporter l'adhésion du lecteur.

La mémoire qu'offre Google? Peut-être n'est-ce qu'un déluge de souvenirs, de sources souvent inconnues, taxant fortement notre capacité d'attention au point de nous engluer dans un océan de fragments informationnels. La mémoire de Google? Partout et instantanément disponible (ou presque), elle fonctionne désormais comme une prothèse incontournable de la mémoire humaine, tout comme l'écriture du dieu Thot.

26. Cette question a suscité une excellente étude : Viktor Mayer-Schönberger, Delete: The Virtue of Forgetting in the Digital Age, Princeton, Princeton University Press, 2011.

27. Mayer-Schönberger, 2011, p. 17.

28. Il s'agit de la nouvelle «Funes, el memorioso » de Borges que Mayer-Schönberger mentionne, ibid., p. 12.

29. Ibid., p. 21. 
Reste enfin une dimension de cette mémoire Google qu'il ne faut pas négliger : la possibilité de retrouver toutes sortes de «faits » sur n’importe qui, n’importe quel événement, n’importe où. Cette étrange mémoire recouvre effectivement les caractéristiques d'un dispositif optique et nous ramène ainsi aux remarques accompagnant le début de ce texte: il s'agit en fait d'un univers panoptique à la fois spatial et temporel ${ }^{30}$. On déborde là les cadres classiques de la mémoire collective pour entrer dans les officines de police, surtout de polices secrètes, tenant sur chacun de nous un dossier extraordinairement complet, un dossier monté pour préparer l'inculpation possible à tout moment. Nous venons de basculer dans l'univers de Kafka et celui de tous ses référents: KGB, STASI, CIA...

诤 弥

Le but de ce petit tour d'horizon n'était pas d'offrir une critique de Google. $\mathrm{Ne}$ viser que cet objectif relèverait d'ailleurs en partie de l'hypocrisie: sans Google, bien des éléments de ce texte n'auraient pu être mobilisés. Il faut donc rendre à César ce qui est à César et convenir de l'immense utilité de ce nouveau dispositif mémoriel qui nous environne désormais, et va nous environner de plus en plus étroitement dans les années à venir. Mais c'est précisément par le biais de l'utilité que la tyrannie s'instaure souvent: cette capacité de ficher toujours plus précisément et exactement toujours plus de détails de la vie de chacun d'entre nous constitue un immense danger. Rappelons que la rafle du Vel'd'Hiv des 16 et 17 juillet 1942, à Paris, n'aurait pas été aussi sinistrement efficace sans la méticulosité de la bureaucratie française. Changent les conditions politiques d'un exercice de traçage et changent alors les dangers et périls découlant de tant de savoir accumulé et ordonné. De plus, la nature de ce que «je» recherche est engrangé et permet de recomposer «mes» intérêts de façon à me servir des produits de consommation susceptibles de «me» tenter: cette simple procédure par échantillonnage s'applique aussi bien au politique qu'à l'économique. De l'identification de mes désirs de consommation à l'interpellation par des pouvoirs hostiles à certaines tendances politiques, il n'y a qu'un pas relativement facile à franchir car déjà résolu sur le plan technique. Seules des lois et des règles éthiques distinguent encore (un peu) citoyens et consommateurs, et protègent (un peu) les premiers; mais les débats en Europe sur la protection des données personnelles face à Google montrent bien que les termes en présence ne relèvent 
pas d'évidences, mais sont plutôt dictés par des objectifs économiques et politiques $^{31}$. Même les démocraties dites libérales diffèrent sur cette question...

Cela dit, ce texte ne cherche pas non plus à grossir la menace panoptique que pourrait constituer Google. Plus fondamentalement, il cherche à montrer comment la mémoire fonctionne dans une articulation complexe entre paramètres personnels et paramètres de groupe, communautaires ou collectifs. Un aspect du trompe-l'œil de Google consiste principalement à nous laisser penser que rien ne change, si ce n'est que tout est plus facile et rapide. La solution technique Google s'offre comme neutre, parfaitement instrumentale, et totalement au service de l'individu qui veut s'en prévaloir. Mais la possibilité de glissements inattendus et de déplacements délétères dans les enjeux de la mémoire demeure réelle. C’est pour cela que l'analogie avec l'analyse platonicienne de l'écriture est intéressante car, dans ses tensions mêmes, elle montre que l'écriture, tout comme Google, transforme la relation de l'individu à son monde, tout en révélant l'importance de ce dispositif pour la vie en commun. De ce point de vue, Google intervient à un niveau extrêmement fondamental qui peut être décrit comme suit: à l'époque de Platon, l'écriture commençait à révéler tout son potentiel largement grâce au développement d'un alphabet complet avec voyelles et consonnes. Instrument puissant de préservation, l'écriture abrite également un désir de naviguer dans la mémoire. Du codex et son organisation aux bibliothèques, ce même désir subsiste. Google, en somme - et prenons ici Google comme emblème de solution -, résout en gros le problème de la récupération de ce qui est contenu dans l'écriture mais, ce faisant, le transmute en données, en «faits». L'écriture, outil fondamental du philosophe pour organiser la vie de la polis, engendre un glissement dans la signification de ce que connaître veut dire. Bacon y reconnaîtrait probablement une menace pour sa Nouvelle Atlantide.

Le roi Thamus, dans sa sagesse, tenterait sans doute de nous rassurer sur le fait que Google, côté connaissances, ne peut que redonner ce que l'écriture livre, et que, côté social et collectif, il déconnecte ces connaissances de pratiquement toute communauté vivante pour en faire le produit d'un algorithme statistique qui sera peut-être soumis, un jour, à l'examen critique de nouvelles communautés. En attendant, donner à penser que le contenu signifiant de l'écriture peut se ramener à des données récupérables constitue un trompe-l'œil d'importance sur lequel il importe de méditer.

31. Eric Pfanner, «In Europe, Challenges for Google», The New York Times, 2 février 2010 , www.nytimes.com/2010/O2/o2/technology/companies/ozgoogle.html (dernière consultation le 10 février 2012). 